\title{
Philosophiques
}

\section{Julien J. Lafontant. Montesquieu et le problème de l'esclavage dans L'Esprit des lois. Éditions Naaman, Sherbrooke, 1979, 164} p.

\section{Josiane Boulad Ayoub}

Volume 8, numéro 2, octobre 1981

URI : https://id.erudit.org/iderudit/203174ar

DOI : https://doi.org/10.7202/203174ar

Aller au sommaire du numéro

Éditeur(s)

Société de philosophie du Québec

\section{ISSN}

0316-2923 (imprimé)

1492-1391 (numérique)

Découvrir la revue

Citer ce compte rendu

Boulad Ayoub, J. (1981). Compte rendu de [Julien J. Lafontant. Montesquieu et le problème de l'esclavage dans L'Esprit des lois. Éditions Naaman, Sherbrooke, 1979, 164 p.] Philosophiques, 8(2), 349-355. https://doi.org/10.7202/203174ar d'utilisation que vous pouvez consulter en ligne.

https://apropos.erudit.org/fr/usagers/politique-dutilisation/ 


\section{COMPTES RENDUS}

Julien J. LAFONTANT. Montesquieu et le probleme et l'esclavage dans L'Esprit des lois. Éditions Naaman, Sherbrooke, 1979, 164 p.

par Josiane Boulad Ayoub

Montesquieu et le probleme de l'esclavage dans L'Esprit des lois est une thèse de doctorat présentée en 1976 par M. Julin Lafontant, de Port-au-Prince, à l'Université d'État de New York à Binghamton. Analysant surtout le livre XV de l'Esprit des Lois, et, en particulier, son chapitre $V$, l'auteur voudrait battre en brèche l'image d'un Montesquieu anti-esclavagiste et montrer que les intérêts économiques et politiques de ce dernier, qui recouperaient par ailleurs ceux de son gouvernement, étaient incompatibles avec la dénonciation du "véritable esclavage», celui des nègres.

Dans ses chapitres 1 et 2 , l'auteur souligne la précipitation, qu'il affirme volontaire, de Montesquieu, quand celui-ci traite de l'origine du droit de l'esclavage à Rome et en Amérique: il critique la signification que le philosophe tire des documents à sa disposition; ce qu'il retient des faits eux-mêmes comme ceux qu'il choisirait de méjuger. Lafontant reproche surtout à Montesquieu, c'est le nerf du chapitre 3, l'arbitraire de la relation établie entre gouvernement despotique et esclavage. Il soutient que l'analyse, à ce sujet, du président du parlement de Bordeaux serait davantage dictée par sa rancoeur devant les mesures prises par Pierre le Grand, par exemple contre la noblesse dont il se sent solidaire, que par la lutte sincère contre l'absolutisme. Or, sur ce point crucial de sa thèse, l'auteur nous semble procéder assez sommairement. Tout d'abord il ne rend pas compte d'un effet objectif entraîné précisément par la politique du despotisme éclairé: le renforcement du servage en Russie, au cours du $18^{\mathrm{e}}$ siècle, sous les régimes de Pierre le Grand puis de Catherine II. Ensuite et surtour il néglige d'affronter, avec l'ampleur et les nuances nécessaires, la critique par Montesquieu du despotisme à son contrepoids, le principe fondamental dans l'Esprit des Lois, de la «séparation des pouvoirs» pour sauvegarder les libertés individuelles et la liberté politique dans un État. Que le parti-pris «féodiste» de Montesquieu, déjà décelé au $18^{\mathrm{e}}$ siècle par Voltaire et Helvétius, avant Althusser dont le Montesquieu figure parmi les ouvrages cités dans la bibliographie de Lafondant, se cache sous le libéralisme souriant du chercheur et de l'écrivain, est certes indiscutable. Cependant, il aurait valu la peine de s'attarder sur les enjeux politiques de ces contradictions et d'analyser comment les raisonnements et les outils scientifiques nouveaux - élaborés par Montesquieu pour maintenir la tradition et dirigés, d'une part, contre les menaces populaires, d'autre part, contre l'expansion de la bourgeoisie d'affaires - furent repris et exploités à son profit par la bourgeoisie révolutionnaire, qui, elle, supprimera, entres autres, la traite puis l'esclavage. 
Le chapitre 4 dénonce la faiblesse scientifique de la théorie du climat qui sert à Montesquieu à fonder sa «trilogie», comme dit l'auteur: despotisme; climat chaud; esclavage. Sans nier cette faiblesse, il aurait été plus juste d'ajouter que Montesquieu voulait se distinguer par cette théorie, d'une part des théologiens en laïcisant la politique, d'autre part des penseurs qui n'avaient que des soucis littéraires. Le chapitre 5, un des plus sérieux de l'ouvrage, dépeint le commerce triangulaire au $18^{\mathrm{e}}$ e siècle, ses iniquités, et expose la série de faits qui autorisent à relier les intérêts personnels de Montesquieu à ceux de l'essor colonial et du commerce du «bois d'ébène», c'est-à-dire la traite des nègres. Dans le chapitre 6, Lafontant s'attaque à l'écriture ironique de Montesquieu, relative au travail servile des Noirs et à leur condition at roce; il soutient que cette ironie n'en est pas une, qu'elle ne recouvre tout compte fait qu'une justification déguisée de l'esclavage. C'est l'un des points les plus discutables de l'ouvrage, selon nous, d'autant plus que la critique porte à faux. L'ironie de Montesquieu ou sa concision sont des procédés constants chez lui, des Lettres Persanes à l'Esprit des Lois, dont il connaît les limites, et qu'il n'utilise pas seulement à l'occasion de l'esclavage. «Si j'avais à soutenir le droit que nous avons eu de rendre les nègres esclaves, voici ce que je dirais: les peuples d'Europe ayant exterminé ceux de l'Amérique, ils ont dû mettre en esclavage ceux de l'Afrique, pour s'en servir à défricher tant de terres. Le sucre serait trop cher, si l'on ne faisait travailler la plante qui le produit par des esclaves»... (Esprit des Lois, $X V, 5$ ): la position de Montesquieu en faveur des oppimés et contre l'opresseur est nette, on ne saurait dire que l'ironie la voile. Il ne faut pas confondre procédé et évaluation de son rôle à un moment précis de la conjoncture, la lutte pour l'abolition du servage et de l'esclavage, par exemple. Libre à l'auteur d'en dénoncer, avec raison, le peu d'efficacité, ou encore de s'élever contre la timidité des attaques de Montesquieu et de les comparer désavantageusement à celles d'Helvétius ou de Bernardin de Saint-Pierre, encore qu'il oublie l'abbé Raynal (Histoire des deux Indes) qui va jusqu’à prophétiser la révolte des Noirs et le remplacement du «Code Noir» (de Colbert) par un «Code Blanc»; mais comment éluder le fait de l'ironie? Sa critique en aurait été d'autant plus vigoureuse s'il avait fait la part des conditions historiques, idéologiques et sociales de l'usage qu'en fait Montesquieu. Ce n'est pas par hasard que la campagne contre l'esclavage se réfugie sous l'ironie des écrits avec Montesquieu mais qu'elle se fait plus sarcastique et pathétique avec Voltaire, tout en s'empêtrant dans les contradictions de la bourgeoisie marchande: "quand nous travaillons aux sucreries et que la meule nous attrape le doigt, on nous coupe la main; quand nous voulons nous enfuir, on nous coupe la jambe; je me suis trouvé dans ces deux cas: c'est à ce prix que vous mangez du sucre en Europe" (Candide, chap. 19), et qu'elle gagne en force et en intensité avec Diderot et Rousseau à la fin du siècle.

La conclusion rassemble les chefs d'accusation relevés contre Montesquieu et formule sa condamnation finale: partial, raciste, aveuglé par ses propres intérêts, Montesquieu, bien inférieur à Helvétius ou à Bernardin de Saint-Pierre, loin d'être l'adversaire de l'esclavage des noirs, l'a admis et l'a justifié. Le lecteur se sent pris au dépourvu devant ce type de texte qui 
s'apparente plus au genre du pamphlet ou du tableau d'honneur et d'infamie qu'à la thèse, proprement dite. Il serait en droit de s'attendre, quand il s'agit d'une remise en question d'un des dispositifs théoriques qui dominent le $18^{\mathrm{e}}$ siècle, à une analyse plus rigoureuse, à une méthodologie moins confuse, à une critique davantage étayée. Y a-t-il jamais eu, du reste, de "mythe à faire tomber» (comme l'écrit le préfacier), celui du Montesquieu anti-esclavagiste? Les commentateurs de l'Europe des Lumières s'accordent à relever les contradictions de ce siècle, et plus important, à en étudier la constitution et les effets; de même ils tracent tous, du point de vue historique ou idéologique, l'évolution difficile de la lutte pour l'abolition du servage et de l'esclavage à laquelle Montesquieu n'a donné que le coup d'envoi, sous le mode ironique. C'est Groethuysen qui faisait remarquer, dans la Pbilosophie de la Révolution française, que "Montesquieu s'est borné à étudier les lois propres à chaque État, à comparer entre elles leurs différentes constitutions; il n'a cherché qu'à découvrir par induction, parmi les matériaux que lui fournit l'histoire, les règles générales de certaines formes de législation" (chap. III, Montesquieu). Les révolutionnaires français reprocheront précisément à Montesquieu ce point de vue: il ne s'agit pas de savoir si les lois s'accordent avec telle ou telle forme de constitution mais de savoir si cette forme est juste ou non. En ce sens, Lafontant est «révolutionnaire» lorsqu'il dénonce la préoccupation de Montesquieu de chercher les raisons de ce qui est plutôt que de chercher ce qui doit être. Peut-on conclure d'un fait à sa validité? Cela revient à mettre l'état de fait à la place d'un état de droit. Avec une pareille méthode toutes les erreurs, toutes les absurdités, tous les crimes deviennent, en effet, légitimes. Il aurait été plus efficace, peut-être, d'aborder la lecture de l'Esprit des Lois sous cet angle d'attaque, et d'approfondir les enjeux du parti-pris de Montesquieu, d'en dégager son "esprit», qui est celui de la vérité politique de la noblesse parlementaire, de suivre les contradictions sur le paradigme de la question de l'esclavage, où s'enferre, de façon révélatrice, le libéralisme économique qui devient l'ennemi de cette liberté réelle et concrète des individus et des groupes réclamée par le parti des Philosophes révoltés dans leur sensibilité, lorsque le libéralisme est développé jusqu'au bout et appliqué au commerce des esclaves. Le scandale, devant les nécessités d'une production industrielle, est encore aujourd'hui d'accepter cette conséquence extrême et abusive qui est la liberté de l'exploiteur de contraindre les nouveaux esclaves à travailler pour lui («c'est à ce prix que nous mangeons du sucre, du chocolat, du café»!) et de continuer théoriquement à ne reprendre, «bourgeoisement», que l'opposition traditionnelle de la «liberté» et de la «licence».

Département de philosophie Université du Québec à Montréal 